\title{
AVALIAÇÃO DE LINHAGENS DE CAFEEIROS QUANTO À TOLERÂNCIA AO ALUMÍNIO PELO MÉTODO DO PAPEL-SOLUÇÃO ${ }^{(1)}$
}

\author{
MARIA DO CARMO LANA BRACCINI ${ }^{(2)}$; HERMINIA EMILIA PRIETO \\ MARTINEZ $^{(3,5)}$; ALESSANDRO DE LUCCA E BRACCINI ${ }^{(4,5)}$ \\ RESUMO
}

\begin{abstract}
Dois experimentos foram desenvolvidos em germinador com o objetivo de se estabelecer um procedimento rápido para seleção de linhagens de cafeeiros tolerantes ao $\mathrm{Al}$, determinar a concentração desse elemento na solução e avaliar a tolerância de 26 linhagens de café ao $\mathrm{Al}$, utilizando o método do papel-solução. No primeiro experimento, sementes de duas linhagens de café foram postas a germinar em presença de diferentes concentrações de $\mathrm{Al}\left(0,25,35\right.$ e $\left.65 \mathrm{mg} \cdot \mathrm{L}^{-1}\right)$ utilizando-se a técnica do papel-solução, e medindo-se o comprimento da raiz principal, após quarenta dias. A linhagem UFV 2237 mostrou-se mais sensível ao Al, comparada à UFV 3880, e a concentração de $35 \mathrm{mg} . \mathrm{L}^{-1}$ permitiu discriminar as linhagens quanto à tolerância. No segundo experimento, sementes de 26 linhagens de café foram colocadas para germinar na ausência e na presença de $\mathrm{Al}$, na concentração de $45 \mathrm{mg} . \mathrm{L}^{-1}$ utilizando-se a técnica do papel-solução. O comprimento da raiz principal foi medido após quarenta e dois dias. As linhagens foram agrupadas em quatro classes de tolerância, pela análise de agrupamento, em função do percentual de redução no comprimento da raiz principal. A elevada diversidade genética apresentada pelas linhagens, quanto à tolerância ao $\mathrm{Al}$, pode ser útil em programas de melhoramento do cafeeiro.

Palavras-chave: café, alumínio, tolerância, técnica de seleção.
\end{abstract}

\section{ABSTRACT \\ SCREENING COFFEE GENOTYPES FOR ALUMINUM TOLERANCE USING THE SOLUTION-PAPER METHOD}

Two experimental trials have been carried out in germination chambers to check, at the seedling stage, coffee plant reaction to the presence of aluminum; the first has been set up to determine the $\mathrm{Al}$ concentration that would best differentiate the genotypes under study while, in the second, twenty-six genetic materials have been tested at the $\mathrm{Al}$ concentration previously defined, as to their reactions to the stress. In the first experiment, seeds of two genotypes (UFV 2237 and UFV 3880) were germinated in the presence of increasing $\mathrm{Al}$ levels $\left(0,25,35\right.$ and $\left.65 \mathrm{mg} . \mathrm{L}^{-1}\right)$, using the solution-paper method, and lengths of primary roots measured after 40 days. The best differentiation was achieved at $35 \mathrm{mg} \cdot \mathrm{L}^{-1} \mathrm{Al}$ concentration, in which UFV 2237 revealed to be more sensitive than UFV 3880. In the second, also using the solution-paper method and a $45 \mathrm{mg} \cdot \mathrm{L}^{-1}$ aluminum level, lengths of primary roots were

$\left(\begin{array}{l}1 \\ { }^{2}\end{array}\right)$ Recebido para publicação em 18 de janeiro de 1999 e aceito em 26 de setembro de 2000.

$\left(^{2}\right)$ Centro de Ciências Agrárias/Universidade Estadual do Oeste do Paraná (UNIOESTE), Rua Pernambuco 1.777, 85960-000 Marechal Cândido Rondon (PR). E-mail: mclana@unioeste.br

( ${ }^{3}$ ) Departamento de Fitotecnia/Universidade Federal de Viçosa (UFV), 36571-000 Viçosa (MG).

$\left(^{4}\right)$ Departamento de Agronomia/Universidade Estadual de Maringá (UEM), Av. Colombo 5.790, 87020-900 Maringá (PR). E-mail: albraccini@uol.com.br

$\left(^{5}\right)$ Com bolsa de produtividade em pesquisa do CNPq. 
measured after 42 days. The results allowed the classification of the twenty-six coffee genotypes into four different groups, based on primary root length percentage reductions, through $\mathrm{Al}$ reaction comparisons, for each genotype, in the presence and absence of stress. The high genetic diversity present strongly indicates the future success, in the selection for this character, in plant breeding programs.

Key words: coffee, aluminum, tolerance, screening.

\section{INTRODUÇÃO}

A produção de café em solos ácidos pode ser reduzida por altas concentrações de alumínio trocável e/ou baixa saturação por bases (PAVAN e Bingham 1982). Na maioria das vezes, o problema é contornado com a aplicação de calcário no solo, apesar de causar o comprometimento da correção da toxicidade de $\mathrm{Al}$ nos horizontes inferiores. A alternativa que oferece maiores possibilidades de sucesso é a correção superficial do solo, juntamente com a utilização de espécies e/ou variedades tolerantes à toxicidade de Al. Muitos pesquisadores têm relatado que espécies e linhagens de plantas diferem amplamente quanto à tolerância ao estresse de Al (FURLANI e CLARK, 1981; FOY, 1988), e que a utilização de plantas tolerantes ao $\mathrm{Al}$ pode ser uma alternativa vantajosa em face dos altos custos das práticas de manejo convencionais (HowELER, 1987).

Encontra-se na literatura grande número de publicações sobre toxicidade de $\mathrm{Al}$ em diversas espécies de interesse econômico; entretanto, é escasso o número de trabalhos com o cafeeiro. Estudos realizados por PaVan e Bingham (1982), Londoño e Valencia-AristizÁbal (1983), PASSO e Ruiz (1995), Rodrigues (1997) e BRACCINI et al. (1998) mostraram que o Al diminui a absorção de nutrientes, o crescimento e desenvolvimento do sistema radicular e da parte aérea, e que as linhagens se comportam diferentemente, quanto à tolerância ao $\mathrm{Al}$.

A redução no crescimento radicular pode ser causada por diferentes formas de alumínio, cuja identificação por meio dos métodos analíticos tradicionais é trabalhosa; além disso, a formação de complexos orgânicos de $\mathrm{Al}$ pode afetar a toxicidade desse metal. Dessa forma, a utilização de testes biológicos é uma alternativa mais conveniente para avaliar a fitotoxicidade ao Al. Ritchey et al. (1988) descreveram dois testes relativamente simples, baseados no crescimento radicular de plântulas de trigo e sorgo, com a finalidade de identificar, tanto a deficiência de $\mathrm{Ca}$ como a toxicidade de $\mathrm{Al}$ em diferentes solos. Os resultados foram obtidos em apenas quatro dias após o transplantio. AHLRichs et al. (1990) utilizaram, também, um método simples e rápido para avaliar diferentes subsolos quanto à toxicidade de $\mathrm{Al}$, com base no crescimento do sistema radicular de trigo e sorgo em placas de Petri, em apenas dois dias. Segundo os pesquisadores, a vantagem do método é que o crescimento da raiz depende apenas da metabolização das substâncias de reserva contidas nas sementes, além do Ca solúvel e da água presentes no solo.

WHEELER et al. (1993) compararam cinco técnicas para avaliar a tolerância de linhagens de trigo em solução nutritiva: por meio do crescimento de raiz, da coloração com hematoxilina após três dias de exposição ao $\mathrm{Al}$, da produção de biomassa seca da parte aérea e raízes, do comprimento de raiz e da observação visual dos danos causados pelo $\mathrm{Al}$ após quatro semanas de crescimento. Dentre os métodos avaliados, a coloração com hematoxilina e a observação visual foram os mais eficientes na identificação das plantas tolerantes e das sensíveis.

A maioria dos métodos disponíveis para selecionar linhagens quanto à tolerância ao Al é baseada em observações do crescimento das raízes em solução nutritiva. Entretanto, existe a necessidade de rigoroso controle do $\mathrm{pH}$ para manter constante a concentração de Al durante o teste. Para contornar esse problema, KONZAK et al. (1976) utilizaram com sucesso o método do papel-solução com base em medidas de comprimento da raiz para avaliar a tolerância ao $\mathrm{Al}$ em plantas de trigo, cevada, arroz, sorgo, milho e soja. As vantagens desse procedimento estão relacionadas à facilidade no controle do $\mathrm{pH}$, pelo uso de uma solução nutritiva tamponada, e à sua simplicidade e rapidez. Portanto, a escolha de características fáceis de serem avaliadas e suficientemente sensíveis para detectar diferenças na tolerância da planta, assim como a utilização de métodos que possibilitem a seleção de grande número de espécies e/ou linhagens, em pequeno espaço físico e curto período de tempo, tornam-se altamente relevantes.

Considerando-se que o café é cultura perene e apresenta taxa de crescimento muito baixa há necessidade de se desenvolverem testes mais simples e rápidos de avaliação, quanto à tolerância ao alumínio. Além disso há poucas informações a respeito da tolerância de linhagens de café ao $\mathrm{Al}$.

Os objetivos deste trabalho foram: 1) avaliar um procedimento rápido para a seleção de linhagens de café quanto à tolerância ao $\mathrm{Al}$;2) determinar a concentração desse elemento na solução que melhor dis- 
crimine as linhagens, quanto à tolerância; e 3) avaliar a tolerância de 26 linhagens de café ao Al.

\section{MATERIAL E MÉTODOS}

Um primeiro ensaio foi conduzido com a finalidade de determinar a concentração de $\mathrm{Al}$ que melhor discriminasse as linhagens de café, quanto à tolerância a esse elemento, utilizando-se o método do papelsolução (KonZAK et al., 1976). O ensaio foi conduzido em germinador, sob temperatura constante de $30 \pm 1{ }^{\circ} \mathrm{C}$. O delineamento foi inteiramente casualizado, com quatro repetições, sendo cada parcela constituída de rolo de papel-toalha do tipo germitest contendo 25 plântulas. Os tratamentos foram distribuídos no esquema fatorial $4 \times 2$, quatro concentrações de $\mathrm{Al}\left(0,25,35\right.$ e $65 \mathrm{mg} . \mathrm{L}^{-1}$ de $\mathrm{Al}$ na forma de $\mathrm{AlCl}_{3}$ ) e de duas linhagens de café, UFV 2237 (Catuaí Vermelho -IAC-15) e UFV 3880 (Catimor -UFV 1603 -232 T15 PN). O potencial osmótico foi mantido constante em todos os tratamentos e igual ao do tratamento com $65 \mathrm{mg} . \mathrm{L}^{-1}$ de $\mathrm{Al}$, utilizando-se polietileno glicol (PEG 6000). As concentrações de PEG foram determinadas pela equação proposta por Michel e KAUFMANN (1973) e corresponderam às seguintes doses, em g.L. $L^{-1}: 25,0 ; 16,6 ; 13,1$ e 0,0 , respectivamente, para os tratamentos $0,25,35$ e $65 \mathrm{mg} . \mathrm{L}^{-1}$ de Al.

Foram semeadas 40 sementes sem o pergaminho entre três folhas de papel-toalha do tipo germitest $(28 \mathrm{~cm} \times 38 \mathrm{~cm})$, previamente umedecidas na soluçãoteste. A composição da solução nutritiva utilizada para embeber o papel foi $\mathrm{MgSO}_{4} 0,1 \mathrm{mmol} \cdot \mathrm{L}^{-1}, \mathrm{KNO}_{3}$ 0,1 mmol.L-1 $\mathrm{NH}_{4} \mathrm{NO}_{3}$ 0,15 mmol. $\mathrm{L}^{-1}$ e $\mathrm{KHC}_{8} \mathrm{H}_{4} \mathrm{O}_{8}$ 8,0 mmol. $\mathrm{L}^{-1}$, além das diferentes concentrações de Al. Devido ao curto período de condução do ensaio, o fósforo foi omitido para evitar precipitação com Al e micronutrientes. As soluções tiveram o $\mathrm{pH}$ corrigido para 4,0, tendo-se utilizado o biftalato de potássio para manter o $\mathrm{pH}$ em torno desse valor durante a condução do ensaio. Para evitar o desenvolvimento de fungos foi adicionado à solução nutritiva o fungicida Captan, a 0,2\%. Os rolos de papel foram colocados na posição vertical, dentro de vasos de plástico com capacidade de $2 \mathrm{~L}$, contendo uma lâmina de $250 \mathrm{~mL}$ de solução nutritiva com as diferentes concentrações de $\mathrm{Al}$, de maneira a manter a base dos rolos sempre umedecida. de acordo com o método descrito por KONZAK et al. (1976). A solução nutritiva foi idêntica à utilizada para embeber o papel germitest, tendo sido reposta semanalmente para manter constante a lâmina de solução. Os vasos foram mantidos no escuro dentro do germinador.

Após 15 dias, os rolos de papel foram abertos e as radículas direcionadas para baixo (posição vertical).
Foram eliminadas as sementes não germinadas ou atacadas por microrganismos, deixando-se 25 plântulas por rolo. Aos 40 dias mediu-se o comprimento da raiz principal, e os resultados foram submetidos à análise de variância e estudo de regressão polinomial, determinando-se a concentração de $\mathrm{Al}$ a ser utilizada no ensaio seguinte.

No segundo ensaio, conduzido no delineamento em blocos casualizados com quatro repetições, sementes de 26 linhagens de café (Quadro 1) foram colocadas para germinar na ausência e presença de $\mathrm{Al}$, na concentração de $45 \mathrm{mg}$. $\mathrm{L}^{-1}$, utilizando-se o método descrito anteriormente. Os tratamentos foram distribuídos no esquema fatorial $26 \times 2$ (linhagens $\mathrm{x}$ níveis de $\mathrm{Al}$ ). $\mathrm{Na}$ ausência de $\mathrm{Al}$ a pressão osmótica foi ajustada adicionando-se PEG 6000 na concentração de 18,5 g.L $\mathrm{L}^{-1}$. O comprimento da raiz principal foi medido aos 42 dias. As linhagens foram agrupadas em diferentes classes de tolerância, em razão da redução percentual no comprimento de raiz $\{\% R C R=$ [1-(Crescimento com Al / Crescimento sem Al)] x $100\}$, pela técnica de análise de agrupamento, segundo o método de otimização de Tocher (CRUZ e ReGAzZI, 1994). Utilizou-se como medida de dissimilaridade, para o agrupamento das linhagens, a distância euclidiana. A dissimilaridade entre linhagens foi representada em gráfico de dispersão, em espaço bidimensional, sendo as coordenadas estimadas de acordo com Cruz e Viana (1994).

\section{RESULTADOS E DISCUSSÃO}

$\mathrm{O} \mathrm{Al}$ afetou o comprimento da raiz principal e o desenvolvimento das raízes laterais, que apresentaram engrossamento e amarelecimento das pontas. Os sintomas de toxicidade de $\mathrm{Al}$ observados em cafeeiro coincidem com os relatados por PAVAN e BINGHAM (1982) e BRACCINI et al. (1998).

O primeiro ensaio revelou que a linhagem UFV 2237 mostrou-se mais sensível ao Al do que UFV 3880, apresentando redução mais acentuada no comprimento de raiz, devido às concentrações de $\mathrm{Al}$ (Figura 1). A mesma tendência foi constatada por BRACCINI et al. (1998).

Houve diferença significativa para linhagens, níveis de $\mathrm{Al}$ e para a interação $\mathrm{Al} x$ linhagens. A partir daí efetuaram-se os desdobramentos nos quais o modelo linear foi significativo para ambas as linhagens. Na concentração de $35 \mathrm{mg} \cdot \mathrm{L}^{-1}$ foi possível separar as linhagens, quanto à tolerância ao $\mathrm{Al}$, uma vez que nas demais concentrações não houve diferença significativa no comprimento da raiz principal das linhagens. Concentrações muito elevadas ou muito baixas desse elemento tendem a eliminar diferenças entre as linha- 
Quadro 1. Identificação e origem das linhagens de café utilizadas no experimento

\begin{tabular}{|c|c|c|}
\hline Identificação & $\begin{array}{l}\text { Origem do } \\
\text { Material }\end{array}$ & Variedade/Híbrido \\
\hline 01 -UFV 2144 & IAC -44 & Catuaí Vermelho \\
\hline 02 -UFV 2145 & IAC -81 & Catuaí Vermelho \\
\hline 03 -UFV 2147 & IAC -99 & Catuaí Vermelho \\
\hline 04 -UFV 2237 & IAC -15 & Catuaí Vermelho \\
\hline 05 -UFV 2149 & IAC -91 & Catuaí Amarelo \\
\hline 06 -UFV 2150 & IAC 376-4-32 & Mundo Novo \\
\hline 07 -UFV 2163 & IAC 388-17-16 & Mundo Novo \\
\hline 08 -UFV 2164 & IAC 515-3 & Mundo Novo \\
\hline 09 -UFV 1340 & UFV 386-19* & Catimor \\
\hline 10 -UFV 1603 & UFV $395-141^{*}$ & Catimor \\
\hline 11 -UFV 2877 & 2 IV EP21.2 & Catimor \\
\hline 12- UFV 2859 & 124 EP 20.1 & Catimor \\
\hline 13 -UFV 3869 & 202 EP 20.1 & Catimor \\
\hline 14 -UFV 3880 & $232 \mathrm{~T} 15 \mathrm{PN}$ & Catimor \\
\hline 15 -UFV 3092 & IAC 2942 & Icatu Vermelho \\
\hline 16 - UFV 2953 & IAC 4040 & Icatu Vermelho \\
\hline 17 - UFV 2954 & IAC 4042 & Icatu Vermelho \\
\hline 18 - UFV 2955 & IAC 4045 & Icatu Vermelho \\
\hline 19 - UFV 2956 & H 4782 & Icatu Vermelho \\
\hline $20-\mathrm{H} 418-3$ & $\begin{array}{l}\text { UFV } 2143-235 x \\
\text { UFV } 443-01\end{array}$ & $\begin{array}{l}\text { Catuaí Amarelo x } \\
\text { Híbrido de Timor }\end{array}$ \\
\hline 21 -H 418-6 & $\begin{array}{l}\text { UFV } 2143-235 x \\
\text { UFV } 443-01\end{array}$ & $\begin{array}{l}\text { Catuaí Amarelo x } \\
\text { Híbrido de Timor }\end{array}$ \\
\hline 22 -H 464-5 & $\begin{array}{l}\text { UFV } 2190-100 x \\
\text { UFV } 440-22\end{array}$ & $\begin{array}{l}\text { Mundo Novo x } \\
\text { Híbrido de Timor }\end{array}$ \\
\hline $23-\mathrm{H} 484-2$ & $\begin{array}{l}\text { UFV } 2164-193 x \\
\text { UFV } 443-03\end{array}$ & $\begin{array}{l}\text { Mundo Novo x } \\
\text { Híbrido de Timor }\end{array}$ \\
\hline 24 -UFV 534 & CIFC 19/1 & Caturra Vermelho \\
\hline 25 -UFV 514 & $\begin{array}{c}\text { Coffea } \\
\text { canephora }\end{array}$ & Guarini \\
\hline 26 -UFV 1359 & UFV $386-45^{*}$ & Catimor \\
\hline
\end{tabular}

*Geração F3 de CIFC HW26/5 (CIFC 19/1 - Caturra Vermelho x CIFC 832/1 - Híbrido de Timor).

gens. Considerando-se que a concentração de $35 \mathrm{mg} \cdot \mathrm{L}^{-1}$ era muito próxima daquela em que não houve discriminação das linhagens ( $\left.35 \mathrm{mg} . \mathrm{L}^{-1}\right)$, optouse pela concentração de $45 \mathrm{mg} . \mathrm{L}^{-1}$. De acordo com MACEDO et al. (1997), um dos principais problemas encontrados na seleção de plantas quanto à tolerância ao Al seria ajustar o nível de estresse de forma a produzir acentuada redução no crescimento dos genótipos sensíveis, limitando, porém, os efeitos sobre

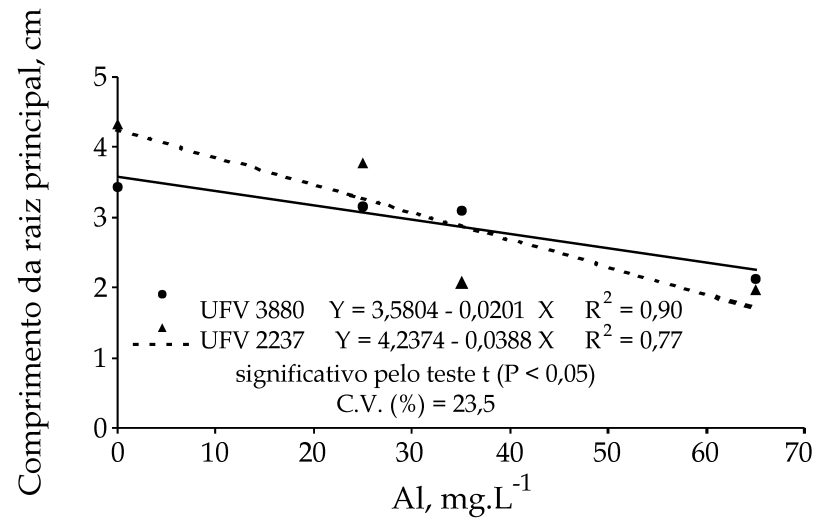

Figura 1. Comprimento da raiz principal das plântulas de duas linhagens de café, em função de concentração de alumínio em papel-solução.

o crescimento dos genótipos tolerantes. KONZAK et al. (1976) verificaram que a concentração de Al requerida para produzir efeitos tóxicos em plântulas de soja, pela técnica do papel-solução, foi aproximadamente dez vezes maior que a utilizada em solução nutritiva.

A raiz principal das 26 linhagens de café apresentou comprimento variando de $2,58 \mathrm{~cm}$ a $4,49 \mathrm{~cm}$, na ausência de $\mathrm{Al}$, e de $1,38 \mathrm{~cm}$ a $4,10 \mathrm{~cm}$, na presença desse elemento, atingindo um percentual de redução no comprimento, de até 53,6\% (Quadro 2). Foi constatado, em quatro linhagens de café, pequeno aumento no crescimento do sistema radicular com a adição de Al.

NuERNBERG et al. (1990) salientaram que o comprimento absoluto de raiz não deve ser utilizado para procedimentos de seleção, uma vez que não permite separação clara entre cultivares tolerantes e sensíveis, bem como entre a biomassa seca da parte aérea e das raízes. Dessa forma, os valores de comprimento de raiz foram expressos em percentagem de redução ou aumento causado pelo Al.

O método de otimização de Tocher (CRUZ e REGAZZI, 1994) permitiu reunir as linhagens de café em quatro classes distintas, quanto à tolerância ao $\mathrm{Al}$, em função do percentual de redução no comprimento da raiz principal (Quadro 3). A classe sensível foi composta por quatro linhagens que apresentaram percentual médio de redução de 49,1\% no comprimento da raiz. A linhagem IAC 4045 apresentou um aumento de $15,9 \%$ no comprimento de raiz, constituindo a classe tolerante. Foram incluídas 18 linhagens na classe moderadamente tolerante por apresentarem redução média de $9,9 \%$ no comprimento da raiz principal, enquanto a classe moderadamente sensível foi constituída por apenas três linhagens, com redução média de $26,8 \%$.

Na figura 2 estão representados o agrupamento e a dispersão gráfica das 26 linhagens de café, com base 
Quadro 2. Comprimento da raiz principal de plântulas de 26 linhagens de café e percentual de variação no comprimento das raízes, em resposta a duas concentrações de alumínio

\begin{tabular}{|c|c|c|c|}
\hline \multirow[t]{2}{*}{ Linhagem } & \multicolumn{2}{|c|}{ Concentração de $\mathrm{Al}\left(\mathrm{mg} . \mathrm{L}^{-1}\right)$} & \multirow[t]{2}{*}{ Variação } \\
\hline & 0 & 8 & \\
\hline & & - & $\%$ \\
\hline 01 -UFV 2144 & 3,81 & 3,40 & $-10,57$ \\
\hline 02 -UFV 2145 & 4,41 & 3,62 & $-17,81$ \\
\hline 03 -UFV 2147 & 3,68 & 3,78 & $+2,92$ \\
\hline 04 -UFV 2237 & 4,26 & 3,77 & $-11,44$ \\
\hline 05 -UFV 2149 & 4,49 & 3,82 & $-14,87$ \\
\hline 06 -UFV 2150 & 4,34 & 3,13 & $-27,84$ \\
\hline 07 -UFV 2163 & 3,40 & 2,72 & $-20,12$ \\
\hline 08 -UFV 2164 & 3,70 & 3,11 & $-15,95$ \\
\hline 09 -UFV 1340 & 3,99 & 4,07 & $+1,88$ \\
\hline 10 -UFV 1603 & 3,01 & 2,50 & $-17,00$ \\
\hline 11 -UFV 2877 & 3,87 & 1,79 & $-53,59$ \\
\hline 12-UFV 2859 & 2,58 & 2,09 & $-18,72$ \\
\hline 13 -UFV 3869 & 2,97 & 1,38 & $-53,61$ \\
\hline 14 -UFV 3880 & 3,42 & 1,95 & $-42,98$ \\
\hline 15 -UFV 3092 & 3,83 & 3,37 & $-11,89$ \\
\hline 16 - UFV 2953 & 3,85 & 3,64 & $-5,33$ \\
\hline 17 - UFV 2954 & 4,41 & 3,36 & $-23,78$ \\
\hline 18 - UFV 2955 & 3,54 & 4,10 & $+15,88$ \\
\hline 19 - UFV 2956 & 3,70 & 3,69 & $-0,07$ \\
\hline $20-\mathrm{H} 418-3$ & 4,04 & 3,24 & $-19,85$ \\
\hline $21-\mathrm{H}$ 418-6 & 3,51 & 3,65 & $+4,13$ \\
\hline $22-\mathrm{H} 464-5$ & 3,97 & 3,85 & $-2,84$ \\
\hline 23 -H 484-2 & 3,13 & 2,63 & $-15,75$ \\
\hline 24 -UFV 534 & 4,20 & 2,26 & $-46,07$ \\
\hline 25 -UFV 514 & 4,24 & 3,02 & $-28,86$ \\
\hline 26 -UFV 1359 & 3,21 & 3,04 & $-5,30$ \\
\hline Média & 3,75 & 3,12 & $-17,86$ \\
\hline
\end{tabular}

nas coordenadas estimadas pela matriz de dissimilaridade, que indica as distâncias entre os grupos. $\mathrm{O}$ grupo I apresenta maior distância em relação ao grupo IV, indicando, portanto, maior divergência genética, ao passo que os grupos II e III apresentam a menor distância intergrupo, com menor divergência genética quanto à tolerância ao $\mathrm{Al}$.

RITCHEY et al. (1988) avaliaram a toxicidade de $\mathrm{Al}$ em diferentes solos, utilizando um cultivar de trigo sensível ao Al. Foi comparado o crescimento radicular na amostra de solo tratada com carbonato de cálcio

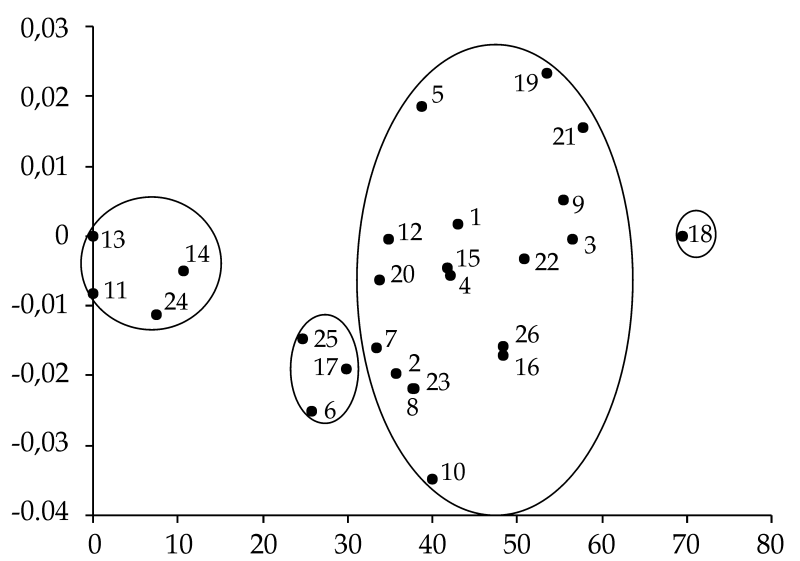

Figura 2. Dispersão de 26 linhagens de café, em função do percentual de variação do comprimento da raiz principal, por meio da projeção de distâncias no plano com base na distância euclidiana.

Quadro 3. Grupos com comportamentos similares estabelecidos pelo método de otimização de Tocher com base na distância euclidiana média (em função da porcentagem de redução do comprimento da raiz principal) de 26 linhagens de café submetidas a dois níveis de alumínio

\begin{tabular}{lccc}
\hline Grupo & $\begin{array}{c}\text { \% de variação } \\
\text { média }\end{array}$ & Classe $^{(1)}$ & Linhagem $^{(2)}$ \\
\hline I & $-49,1$ & S & $11,13,24,14$ \\
II & $-26,8$ & MS & $6,25,17$ \\
III & $-9,9$ & MT & $16,26,22,19$, \\
& & & $9,3,21,1,4$, \\
& & & $15,5,23,8,10$, \\
& & & $2,12,20,7$ \\
IV & $+15,9$ & T & 18 \\
\hline
\end{tabular}

${ }^{(1)}$ S = Sensível; MS = Moderadamente Sensível; MT = Moderadamente Tolerante; $\mathrm{T}=$ Tolerante.

(2) 1 (UFV 2144), 2 (UFV 2145), 3 (UFV 2147), 4 (UFV 2237), 5 (UFV 2149), 6 (UFV 2150), 7 (UFV 2163), 8 (UFV 2164), 9 (UFV 1340), 10 (UFV 1603), 11 (UFV 2877), 12 (UFV 2859), 13 (UFV 3869), 14 (UFV 3880), 15 (UFV 3092), 16 (UFV 2953), 17 (UFV 2954), 18 (UFV 2955), 19 (UFV 2956), 20 (H 418-3), 21 (H 418-6), 22 (H 464-5), 23 (H 484-2), 24 (UFV 534), 25 (UFV 514), 26 (UFV 1359).

para elevar o teor de Ca a $0,1 \mathrm{cmol}_{\mathrm{c} . \mathrm{dm}}{ }^{-3}$ ao crescimento radicular na amostra que recebeu carbonato de cálcio para neutralizar o Al trocável. Quando o crescimento foi inferior a 50\%, a amostra de solo apresentou níveis de $\mathrm{Al}$ considerados tóxicos. Linhagens de café que apresentaram crescimento radicular inferior a $51 \%$ foram classificadas como sensíveis ao Al. O critério adotado neste trabalho é semelhante ao encontrado na literatura.

A classe sensível é constituída por três linhagens de Catimor e uma de Caturra Vermelho. O Catimor é 
um cultivar originado de hibridações entre Caturra Vermelho e Híbrido de Timor. Este último participou da origem dos quatro híbridos avaliados no presente trabalho, os quais apresentaram comportamento mais tolerante. Portanto, a grande sensibilidade do Catimor deve-se, provavelmente, à participação do cultivar Caturra em sua origem.

De forma geral, as linhagens de Catuaí e Icatu e os híbridos foram mais tolerantes ao $\mathrm{Al}$, uma vez que apresentaram menor redução no comprimento de raiz.

\section{CONCLUSÕES}

1. As linhagens de café apresentaram acentuadas diferenças quanto à tolerância ao alumínio.

2. O método empregado e o procedimento estatístico adotado permitiram selecionar um grande número de linhagens de café em curto período de tempo e apresentar os resultados de forma resumida e simplificada.

3. A concentração de $45 \mathrm{mg}$. $\mathrm{L}^{-1} \mathrm{de} \mathrm{Al}$, utilizando-se a técnica do papel-solução, foi adequada para detectar diferenças entre linhagens de cafeeiro quanto à tolerância ao Al.

4. As três linhagens de Catimor (UFV 2877, UFV 3869 e UFV 3880) e uma de Caturra Vermelho (UFV 534) foram classificadas como sensíveis ao Al. 'Mundo Novo' (IAC 376-4-32), 'Icatu Vermelho' (IAC 4042) e 'Guarini' (UFV 514) pertencem à classe moderadamente sensível. 'Icatu Vermelho' (IAC 4045) constituiu-se na linhagem mais tolerante ao $\mathrm{Al}$, ao passo que a maioria foi classificada como moderadamente tolerante.

\section{REFERÊNCIAS BIBLIOGRÁFICAS}

AHLRICHS, J.L.; KARR, M.C.; BALIGAR, V.C.; WRIGHT, R.J. Rapid bioassay of aluminum toxicity in soil. Plant and Soil, The Hague, v.122, p.279-285, 1990.

BRACCINI, M.C.L.; MARTINEZ, H.E.P.; PEREIRA, P.R.G.; SAMPAIO, N.F.; SILVA, E.A.M. Tolerância de genótipos de cafeeiro em solução nutritiva: I. crescimento e desenvolvimento da parte aérea e sistema radicular. Revista Brasileira de Ciência do Solo, Viçosa, v.22, n.3, p.435-442, 1998.

CRUZ, C.D.; REGAZZI, A.J. Modelos biométricos aplicados ao melhoramento genético. Viçosa: Imprensa Universitária, 1994. 390p.

CRUZ, C.D.; VIANA, J.M.S. A methodology of genetic divergence analysis based on sample unit projection on two-dimensional space. Revista Brasileira de Genética, Ribeirão Preto, v.17, n.1, p.69-73, 1994.
FOY, C.D. Plant adaptation to acid aluminum-toxic soils. Communications in Soil Science and Plant Analysis, New York, v.19, p.959-987, 1988.

FURLANI, P.R.; CLARK, R.B. Screening sorghum for aluminum tolerance in nutrient solutions. Agronomy Journal, Lancaster, v.73, p.587-594, 1981.

HOWELER, R.H. Effective screening techniques for tolerance to aluminum toxicity. In: Gourley, L.M.; Salinas, J.G. (Eds.). Sorghum for acid soils. Cali: CIAT, 1987. p.173-186.

KONZAK, C.F.; POLLE, E.; KITTRICK, J.A. Screening several crops for aluminum tolerance. In: workshop on plant adaptation to mineral stress in problem soils., 1976, Beltsville. Proceedings. Ithaca: Cornell University Press, 1976. p.311-327.

LONDOÑN, M.E.A; VALENCIA-ARISTIZÁBAL, G. Toxicidad de aluminio en plantas de café. Cenicafé, Bogotá, v.34, n.3, p.61-97, 1983.

MACEDO, C.C.; KINET, J.M.; SINT JAN, V. van. Effects of duration and intensity of aluminum stress on growth parameters in four genotypes differing in aluminum sensitivity. Journal of Plant Nutrition, Athens, v.20, p.181-193,1997.

MICHEL, B.E ; KAUFMANN, M.R. The osmotic potential of polyethilene glycol 6000. Plant Physiology, Lancaster, v.51, p.914-916, 1973.

NUERNBERG, N.J.; BISSANTI, C.A.; CAMPBELL, T.A.; FOY, C.D. Screening pasture plants for aluminum tolerance. In: El Bassam, N.; Dambroth, M.; Loughman, B.C. (Eds.). Genetic aspects of plant mineral nutrition. Wageningen: Kluwer Academic Publishers, 1990. p.203-208.

PASSO, R.R.; RUIZ, H.A. Tolerância dos cafeeiros conilon e catuaí à toxidez causada pelo alumínio e manganês. Revista Ceres, Viçosa, v.42, n.239, p.45-52, 1995.

PAVAN, M.A.; BINGHAM, F.T. Toxicity of aluminum to coffee seedlings in nutrient solution. Soil Science Society American Journal, Madison, v.46, p.993-997, 1982.

RITCHEY, K.D.; SOUSA, D.M.G.; RODRIGUES, G.C. Testes biológicos para diagnóstico da deficiência de cálcio e toxicidade de alumínio em solos. Revista Brasileira de Ciência do Solo, Campinas, v.12, p.113-120, 1988.

RODRIGUES, L.A. Crescimento e composição mineral na parte aérea e nas raízes de duas variedades de café em resposta à calagem na subsuperfície do solo. Viçosa, 1997. 89p. Dissertação (Mestrado em Fitotecnia) - UFV.

WHEELER, D.M.; EDMEADES, D.C.; CRISTIE, R.A.; GARDNER, R. Comparison of techniques for determining the effect of aluminum on the growth of, and the inheritance of aluminum tolerance in wheat. In: Randall, P.J.; Delhaize, E.; Richards, R.A.; Munns, R. (Eds.). Genetic aspects of plant mineral nutrition. Wageningen: Kluwer Academic Publishers, 1993. p.203-208. 